\title{
Perfect absorption and no reflection in disordered photonic crystals
}

\author{
Jin-Hui Wu, ${ }^{1}$ M. Artoni, ${ }^{2}$ and G. C. La Rocca ${ }^{3}$ \\ ${ }^{1}$ Center for Quantum Sciences and School of Physics, Northeast Normal University, Changchun 130117, China \\ ${ }^{2}$ Department of Engineering and Information Technology and INO-CNR Sensor Lab, Brescia University, 25133 Brescia, Italy \\ ${ }^{3}$ Scuola Normale Superiore and CNISM, 56126 Pisa, Italy
}

(Received 13 March 2017; published 26 May 2017)

\begin{abstract}
Understanding the effects of disorder on the light propagation in photonic devices is of major importance from both fundamental and applied points of view. Unidirectional reflectionless and coherent perfect absorption of optical signals are unusual yet fascinating phenomena that have recently sparked an extensive research effort in photonics. These two phenomena, which arise from topological deformations of the scattering matrix $S$ parameters space, behave differently in the presence of different types of disorder, as we show here for a lossy photonic crystal prototype with a parity-time antisymmetric susceptibility or a more general non-Hermitian one.
\end{abstract}

DOI: 10.1103/PhysRevA.95.053862

\section{INTRODUCTION}

Artificially engineered (optical) materials offer an enormous degree of freedom for manipulating (light) waves, as they provide nearly arbitrary variations in the components of their effective permittivity. Among them, unidirectional reflectionless (URL) media [1-3], where reflection from one side is significantly suppressed, and coherent perfect absorption (CPA) media [4-10], where the two-sided incident waves are completely quenched, are extensively studied wave phenomena, especially in optics [6,9-17]. On-chip implementation of URL media, e.g., is expected to underpin a new generation of photonic devices [18], while CPA media clearly provides an additional flexibility to tune absorption when compared to perfect one-port absorbers.

A great number of these applications rely on crystal structures where order and periodicity are essential attributes. Most ubiquitous among them are photonic crystals [19] that exploit order and periodicity on the scale of the wavelength to manipulate the phase and flow of light beams either in the form of a familiar solid distributed Bragg reflector [20] or in the form of a more sophisticated atomic crystal structure [21,22]. Disorder becomes then a detrimental feature that is best avoided or minimized to improve optical performance.

Disorder due, e.g., to the imperfect manufacturing process inevitably exists also in most of the photonic crystals currently being used to implement URL and CPA. Thus it is essential to assess how robust these two phenomena are in the presence of variant types of disorder. Exploiting the fact that coherently driven multilevel atoms have the uncommon advantages of real-time all-optical tunable and reconfigurable capabilities [23-29], we focus here on a realistic atomic photonic crystal structure [30-42] where the effect of disorder on URL and CPA phenomena could be simultaneously assessed.

More specifically, we use a lossy atomic crystal lattice with a $P T$-antisymmetric or more general non-Hermitian susceptibility $[2,43,44]$, known to exhibit both URL and CPA, to assess the robustness of these two phenomena against (i) uncorrelated and (ii) self-correlated random fluctuations in the typical structural or geometric lattice parameters [45]. Disorder archetypes in the width $(d)$ of the atomic density distribution within a unit cell and in the period $(a)$ of the cold-atomic lattice for each unit cell are briefly illustrated in
Sec. II and investigated in Sec. III through numerical computation of the atomic crystal reflectivities and the scattering matrix eigenvalues. The main conclusions of our work are summarized in Sec. IV.

\section{THE MODEL}

\section{A. The photonic crystal}

Our atomic photonic crystal is shown in Fig. 1(a). We consider a one-dimensional (1D) atomic lattice of period $a=0.5 \lambda_{o} / \cos \theta_{o}$, with $\lambda_{o}$ being the wavelength of the red-detuned laser beams forming the dipole traps and $\theta_{o}$ being the angle between the intersecting dipole-trap laser beams [not shown in Fig. 1(a)] and the lattice axis along $\vec{z}$. Within the $j$ th unit cell, the trapped atoms are taken to have a Gaussian density distribution,

$$
\mathcal{N}_{j}(z)=N \frac{1}{\sqrt{2 \pi d^{2}}} \exp \left[-\left(z-z_{j}\right)^{2} / 2 d^{2}\right],
$$

with $N$ being the average density and $d$ the width of the atomic distribution at the $j$ th cell location, $z_{j}=j \times a$. Such atoms are driven into the four-level $N$ configuration by three coherent fields of frequencies (real amplitudes) $\omega_{p}\left(\mathbf{E}_{p}\right)$, $\omega_{c}\left(\mathbf{E}_{c}\right)$, and $\omega_{d}\left(\mathbf{E}_{d}\right)$ [2,43] [see Fig. 1(b)]. The weak probe field $\omega_{p}$, moderate coupling field $\omega_{c}$, and strong dressing field $\omega_{d}$ couple, respectively, the allowed transitions $|1\rangle \leftrightarrow|3\rangle$, $|2\rangle \leftrightarrow|3\rangle$, and $|2\rangle \leftrightarrow|4\rangle$. The corresponding detunings (Rabi frequencies) are $\Delta_{p}=\omega_{p}-\omega_{31}, \Delta_{c}=\omega_{c}-\omega_{32}$, and $\Delta_{d}=$ $\omega_{d}-\omega_{42}\left(\Omega_{p}=\mathbf{E}_{p} \cdot \mathbf{d}_{13} / 2 \hbar, \Omega_{c}=\mathbf{E}_{c} \cdot \mathbf{d}_{23} / 2 \hbar\right.$, and $\Omega_{d}=$ $\mathbf{E}_{d} \cdot \mathbf{d}_{24} / 2 \hbar$ ) with $\omega_{i j}=\omega_{i}-\omega_{j}$ being resonant transition frequencies and $\mathbf{d}_{i j}$ being electric-dipole moments $(i, j=$ 1,2,3).

Under the rotating-wave and electric-dipole approximations, solutions of density matrix equations in the $j$ th cell yield, for a weak-probe susceptibility $\left(\Delta_{c}=0\right)$,

$\chi_{p_{j}}(z)=\frac{\mathcal{N}_{j}(z) d_{13}^{2}}{2 \varepsilon_{0} \hbar} \frac{i\left\{\gamma_{12}-i\left[\Delta_{p}+\delta_{d s}(z)\right]\right\}}{\Omega_{c}^{2}+\left(\gamma-i \Delta_{p}\right)\left\{\gamma_{12}-i\left[\Delta_{p}+\delta_{d s}(z)\right]\right\}}$,

where we have phenomenologically introduced the dephasing rates $\gamma_{i j}$ with the assumption $\gamma_{13}=\gamma_{14}=\gamma \gg \gamma_{12}$. Note that the dressing field comprises a red-detuned traveling-wave 


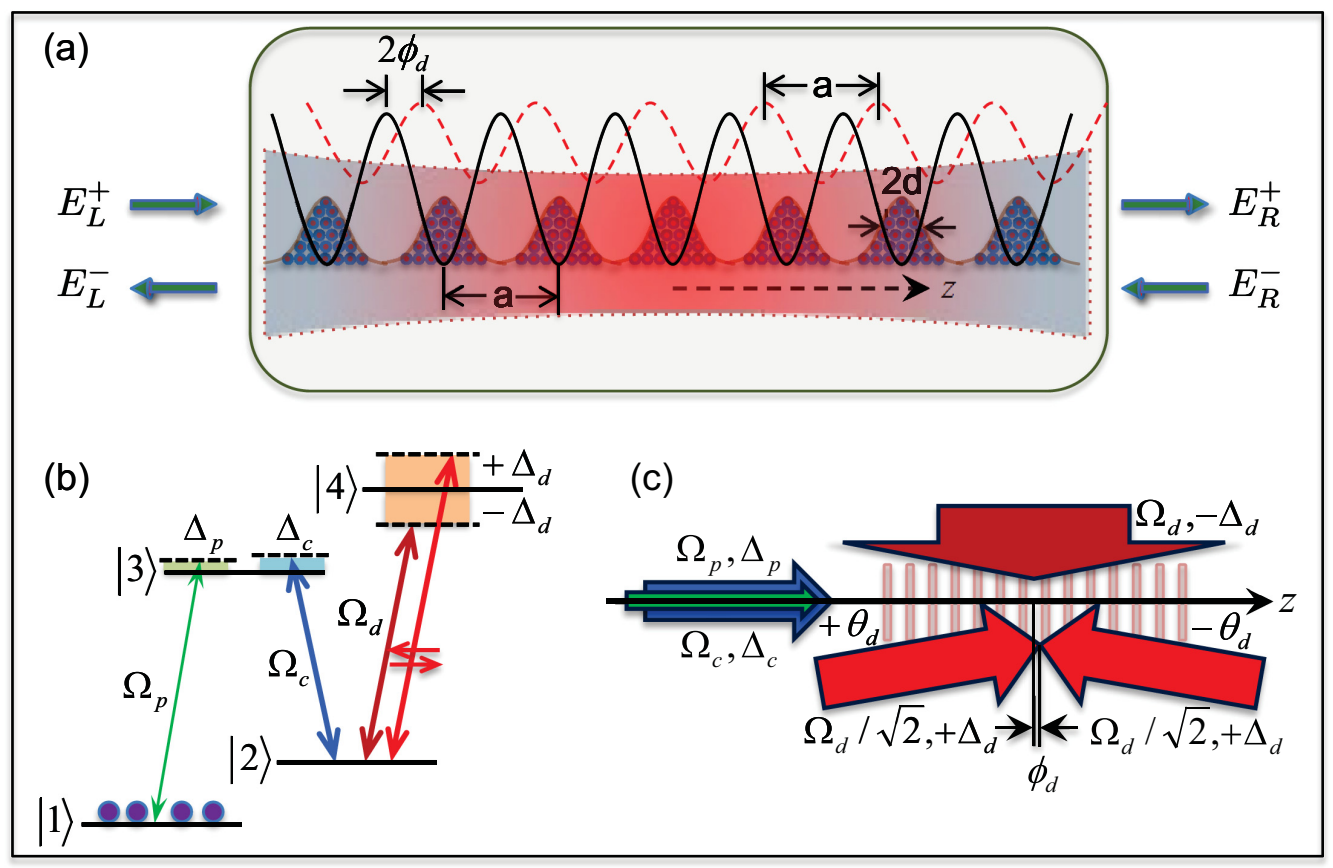

FIG. 1. The atomic photonic crystal. (a) Cold ${ }^{87} \mathrm{Rb}$ atoms, loaded in a $1 \mathrm{D}$ optical lattice (black solid curve) of period $a$ and subject to a three-component dressing field (red dashed curve) as in (c), are used to realize the atomic photonic crystal of Sec. II. The atoms suffer a space-dependent dynamic level shift with the same periodicity, but phase shifted $\left(\phi_{d}\right)$ with respect to the optical lattice. The incident probe electric field amplitudes $\left(E_{R}^{-}, E_{L}^{+}\right)$are scattered by the atomic crystal into the outgoing electric field amplitudes $\left(E_{L}^{-}, E_{R}^{+}\right)$through a process described by the scattering matrix $S$ in Eq. (6). For fields $\left(E_{R}^{-}\right)$incident from the right, e.g., outgoing amplitudes consist of waves $\left(E_{L}^{-}\right)$transmitted with amplitude $t_{R}$ in the $-z$ direction as well as waves $\left(E_{R}^{+}\right)$reflected with amplitude $r_{R}$ in the $+z$ direction, and likewise for fields $\left(E_{L}^{+}\right)$incident from the left and reflected (transmitted) with amplitude $r_{L}\left(t_{L}\right)$; while, in general, $r_{L} \neq r_{R}, t_{L}=t_{R}=t$. URL occurs when either $R_{L}$ or $R_{R}$ vanishes, whereas CPA corresponds to vanishing outgoing fields $\left(E_{L}^{-}=E_{R}^{+}=0\right)$ for a specific incident configuration of fields. (b) The ${ }^{87} \mathrm{Rb}$ atoms are driven into a four-level $N$ configuration by a weak near-resonant probe field (green) on the $|1\rangle \leftrightarrow|3\rangle$ transition, a moderate resonant coupling field (blue) on the $|2\rangle \leftrightarrow|3\rangle$ transition and a strong far-detuned dressing field (red) on the $|2\rangle \leftrightarrow|4\rangle$ transition. (c) The probe, with Rabi frequency $\Omega_{p}$ and detuning $\Delta_{p}$, and the coupling, with Rabi frequency $\Omega_{c}$ and $\Delta_{c}=0$, propagate in the $z$ direction. The dressing field has instead a TW component propagating in the $x$ direction, with detuning $-\Delta_{d}$, and a SW component modulated in the $z$ direction, with detuning $+\Delta_{d}$.

(TW) component and a blue-detuned standing-wave (SW) component so as to yield a small space-dependent dynamic shift [43],

$\delta_{d s}(z)=\frac{\Omega_{d}^{2}}{\Delta_{d}} \cos \left[2\left(k_{d} z-\phi_{d}\right)\right] \equiv \delta_{d 0} \cos \left[2\left(k_{d} z-\phi_{d}\right)\right]$,

for level $|2\rangle\left(\left|\Delta_{d}\right| \gg \Omega_{d}\right)$. Both forward and backward beams of the SW dressing component [see Fig. 1(c)] are allowed to have a small angle $\theta_{d}$ relative to the lattice axis $\vec{z}$ and we can attain $k_{d}=\pi / a$ by modulating the two small angles $\theta_{o}$ and $\theta_{d}$ even if $\lambda_{d} \neq \lambda_{o}$. The shift $\phi_{d}$ is the (phase) mismatch between the optical lattice (black solid curve) and the space-periodic dynamic shift $\delta_{d s}(z)$ (red dashed curve) arising, respectively, from the dipole-trap beams and from the dressing field beams. In particular, for $\phi_{d}= \pm \pi / 4$ and $\Delta_{p}=0, \delta_{d s}(z)$ turns out to be a sine function of $z$, i.e., in quadrature with the atomic density distribution $\mathcal{N}_{j}(z)$ along the optical lattice. In this case, the correlated modulations in $\mathcal{N}_{j}(z)$ and $\delta_{d s}(z)$ result in a $P T$-antisymmetric susceptibility with its real (imaginary) part being an odd (even) function of the position $z[2,43]$. This implies, in particular, both that the system is a pseudo-Hermitian one [46] and that it satisfies the spatial Kramers-Kronig relations [3]. In the case of $\phi_{d} \neq \pm \pi / 4$ or $\Delta_{p} \neq 0$, however, $\chi_{p_{j}}(z)$ will no longer be pseudo-Hermitian.

Although in atomic photonic crystal structures typical probe susceptibilities provide periodic modulations with very small contrasts of refractive index, sizable reflectivities may still be observed, as shown in Fig. 2, due to Bragg reflection for probe wave vectors $k_{p}$ very close to $\pi / a$. This can be attained by small tunings of the probe misalignment angle $\left(\theta_{p}\right)$ with respect to the crystal $z$ axis. Within different parameter regimes than used here, even fully developed photonic stop bands may be obtained [47]. Two entirely different regimes of symmetric Figs. 2(a) and 2(b) and asymmetric [Figs. 2(c) and 2(d)] reflectivities are achieved in the absence or presence of the driving field, respectively. In the former case, the photonic crystal has a mirror symmetry $\left(R_{L}=R_{R}\right)$ associated with a standard electromagnetically induced transparency (EIT)-probe susceptibility ( $\Lambda$ configuration) [48] vanishing, in particular, at two-photon resonance $\left(\Delta_{p}=\Delta_{c}=0\right)$ where we observe zero-reflectivity $\operatorname{dips}\left(R_{L}=R_{R}=0\right)$. In the latter case, we have $R_{L} \neq R_{R}$ with the maximal reflectivities difference occurring surprisingly at $\Delta_{p}=\Delta_{c}=0$. Quite large left-right asymmetries can actually be achieved through optimal tuning of the probe parameters $\left\{k_{p}, \theta_{p}\right\}$, as discussed in details in Sec. III. 

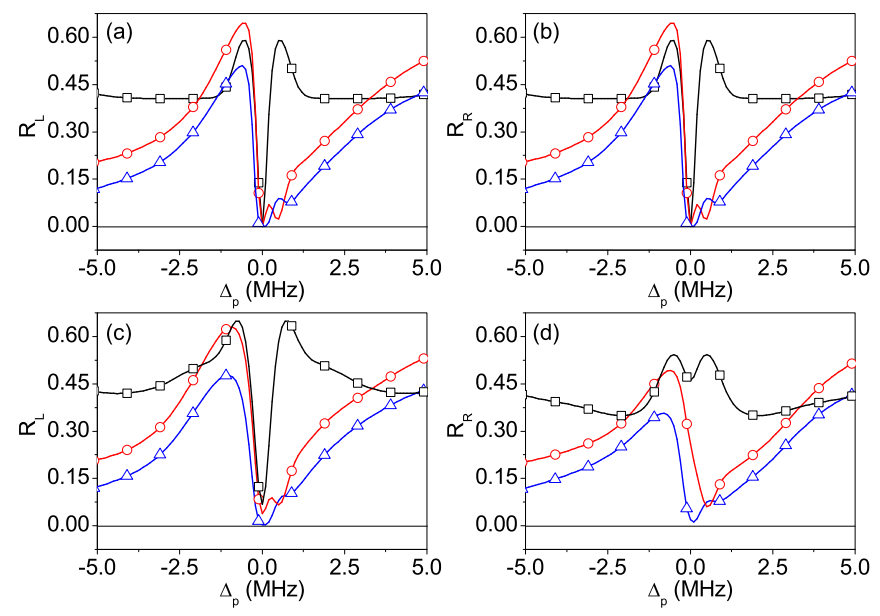

FIG. 2. (a, b) Symmetric reflectivities spectra $\left(R_{L}=R_{R}\right)$ as a function of detuning $\Delta_{p}$ for wave vectors $k_{p}$ close to the Bragg resonance "without" the driving field $\left(\delta_{d 0}=0\right)$. The black-square, red-circle, and blue-triangle curves correspond, respectively, to $\lambda_{p} / \cos \theta_{p}=800.00,799.75$, and $799.50 \mathrm{~nm}$. The lattice parameters are $a=10 d=400 \mathrm{~nm}, \lambda_{o}=800.00 \mathrm{~nm}$, and $\theta_{o}=0^{0}$. (c, d) Asymmetric reflectivities spectra $\left(R_{L} \neq R_{R}\right)$ against $\Delta_{p}$ as above, but "with" the driving field $\left(\delta_{d 0}=1.2 \mathrm{MHz}\right)$ whose parameters are $\lambda_{d}=$ $794.98 \mathrm{~nm}, \theta_{d}=6.4^{0}$, and $\phi_{d}=\pi / 4$. Other parameters suitable to cold ${ }^{87} \mathrm{Rb}$ atoms are $\gamma=3.0 \mathrm{MHz}, \gamma_{12}=0, \Delta_{c}=0, \Omega_{c}=2.5 \mathrm{MHz}$, $d_{13}=2.0 \times 10^{-29} \mathrm{C} \mathrm{m}, L=0.6 \mathrm{~mm}$, and $N=2.0 \times 10^{12} \mathrm{~cm}^{-3}$.

One important application of $P T$-antisymmetric susceptibilities is to realize high-contrast asymmetric reflectivities including fully unidirectional reflection, which one can examine by directly adopting transfer matrix methods to Eq. (2) [47]. To this end, we first derive the $2 \times 2$ unimodular transfer matrix $M_{j}$ of the $j$ th lattice site by dividing the period into, e.g., 100 thin layers of identical thickness $\delta z$ but distinct atomic density $\mathcal{N}_{j}\left(z_{l}\right)$ for $l \in\{1,100\}$. The transfer matrix of such a thin layer is

$$
m_{j}\left(z_{l}\right)=\frac{1}{t_{j}\left(z_{l}\right)}\left[\begin{array}{cc}
t_{j}^{2}\left(z_{l}\right)-r_{j}^{2}\left(z_{l}\right) & +r_{j}\left(z_{l}\right) \\
-r_{j}\left(z_{l}\right) & 1
\end{array}\right],
$$

with the corresponding reflection and transmission complex amplitudes $r_{j}\left(z_{l}\right)$ and $t_{j}\left(z_{l}\right)$ determined by the complex refractive index $n_{p j}\left(z_{l}\right) \simeq 1+\chi_{p_{j}}\left(z_{l}\right) / 2$ [47]. The $j$ th-period matrix is then

$$
M_{j}=m_{j}\left(z_{1}\right) \times \cdots \times m_{j}\left(z_{l}\right) \times \cdots \times m_{j}\left(z_{100}\right),
$$

in terms of which the transfer matrix for a $1 \mathrm{D}$ atomic lattice of length $L=(2 k+1) a$ can be written as

$$
M_{0}^{2 k+1}=M_{-k} \cdots M_{0} \cdots M_{+k} .
$$

From (5), the probe complex amplitudes $t, r_{L}$, and $r_{R}$ at both lattice ends lead to the following expressions for the transmittivity $(T)$ and reflectivities $\left(R_{L, R}\right)$ :

$$
\begin{aligned}
T & =|t|^{2}=\left|\frac{1}{M_{0(22)}^{2 k+1}}\right|^{2}, \quad R_{L}=\left|r_{L}\right|^{2}=\left|\frac{M_{0(12)}^{2 k+1}}{M_{0(22)}^{2 k+1}}\right|^{2}, \\
R_{R} & =\left|r_{R}\right|^{2}=\left|\frac{M_{0(21)}^{2 k+1}}{M_{0(22)}^{2 k+1}}\right|^{2},
\end{aligned}
$$

and to the scattering matrix

$$
S=\left[\begin{array}{cc}
t & r_{L} \\
r_{R} & t
\end{array}\right]
$$

relating the outgoing field amplitudes $\left(E_{L}^{-}\right.$and $\left.E_{R}^{+}\right)$to the incoming field amplitudes $\left(E_{R}^{-}\right.$and $\left.E_{L}^{+}\right)$.

For the specific representation (6) of matrix $S$ [49] with eigenvalues

$$
\lambda_{s}^{ \pm}=t \pm \sqrt{r_{L} r_{R}}
$$

URL occurs when either $r_{L}$ or $r_{R}$ vanishes, corresponding to a non-Hermitian degeneracy [50] of matrix $S$ also known as an exceptional point [51,52], whereas CPA, on the other hand, occurs when

$$
\lambda_{s}^{+}=0 \quad \text { or } \quad \lambda_{s}^{-}=0,
$$

i.e., when $\operatorname{det}(S)=0$ (the latter condition being independent of the $S$-matrix representation chosen).

\section{B. The disorder}

Cold atoms in disordered potentials have been extensively investigated [29,53-57], especially in connection with Anderson localization [58]. Here, we are not interested in the atomic dynamics in random potentials per se, but only in the effects that a small disorder perturbing the periodic atomic distribution may have on the atomic crystal optical response, in much the same way as is done by the random perturbation of a speckle potential when superposed to an ideal optical lattice [58]. Perturbing the ideal periodicity of the real and imaginary parts of the local probe susceptibility $\chi_{p_{j}}(z)$ as well as their phase relation may, in fact, hamper the ideal URL and CPA regimes $[2,44]$ that one attains in the absence of disorder. This is examined in the following by introducing disorder in both geometrical and structural parameters whose random fluctuations are responsible for departures from ideal URL and CPA regimes.

Uncorrelated disorder. We start by considering disorder in the width of the atomic spatial distribution at each lattice site [see Fig. 1(a)]. Such an uncorrelated structural disorder may be modeled by introducing random variations of width $d$ in Eq. (1), which thus become functions of the $j$ th site as

$$
d \rightarrow d_{j}=d+\delta d_{j}=d+d \times \delta_{j} .
$$

Since we do not know a priori how large a random deviation from the average width $d$ should be to observe departures from the ideal URL and CPA regime $[2,44]$, we introduce a disorder-strength parameter. The latter is taken to be the largest variation $\Delta d$ in the width of the Gaussian distribution in Eq. (1) such that

$$
\left|\delta d_{j}\right| \leqslant \Delta d \leqslant d .
$$

Thus, $\left\{\delta_{j}\right\}$ in Eq. (7) represent sequences of uncorrelated random numbers [45] uniformly distributed, respectively, in the interval $[-\Delta d / d, \Delta d / d]$. This source of structural disorder represents how the ideal atomic distribution sketched in the first row of Fig. 3 is affected by uncorrelated random variations in the width of the Gaussian distribution. Such a randomly 


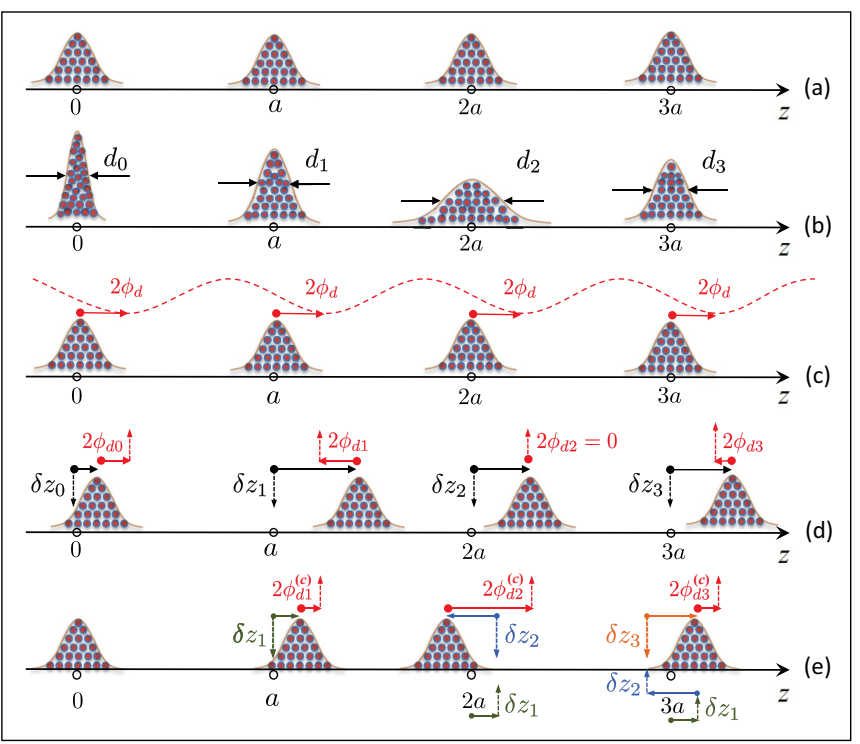

FIG. 3. Disorder models. (a) Schematic atomic distribution of an ideal lattice. (b) Structural disorder entailing "uncorrelated" fluctuations in the atomic spatial distribution width $d_{j}$ [Eq. (7)] at each lattice. (d) Geometric disorder entailing "uncorrelated" fluctuations $\delta z_{j}$ in the site position $z_{j}$ [Eq. (9)] of each lattice and (c) concomitant phase variations $2 \phi_{d_{j}}$ [Eq. (10)] with respect to the lattice site [see Fig. 1(a)]. (e) Geometric disorder entailing "self-correlated" fluctuations of the position $z_{j}^{(c)}$ [Eq. (11)] arising from random variations $\delta z_{j}$ (in color) at all previous site positions along with the concomitant phase mismatch $2 \phi_{d_{j}}^{(c)}$ [Eq. (12)].

perturbed (atomic) photonic lattice is sketched in the second row of Fig. 3.

Lattice disorder may also arise from random perturbation of the potential well position $z_{j}$. Such an uncorrelated geometric disorder is accounted instead by introducing random fluctuations of positions $z_{j}$ in Eq. (1) as

$$
z_{j}=a j+\delta z_{j}=a j+a \zeta_{j}
$$

where $a$ is the lattice period. Similarly to $\left\{\delta_{j}\right\}$ in Eq. (7), here $\left\{\zeta_{j}\right\}$ represent sequences of uncorrelated random numbers uniformly distributed in the interval $[-\Delta a / a, \Delta a / a]$, with $\Delta a \ll a$. It is worth noting that in the setup of Fig. 1, the disorder on the site position is accompanied by a variation of the phase mismatch $\phi_{d}$, which in turn becomes a function of positions $z_{j}$,

$$
\phi_{d_{j}}=\phi_{d}-\frac{\pi}{a} z_{j}=\phi_{d}-\pi \zeta_{j}
$$

this is to be intended as a specific form of fluctuation of the phase mismatch, determined by the disorder in site positions leading to the randomly perturbed lattice sketched in the third and fourth row of Fig. 3.

Self-correlated disorder. We then consider a model of selfcorrelated disorder where the $j$ th-site position $z_{j}$ is affected by the random variations of the positions of all previous sites as

$$
z_{j}^{(c)}=a j+\sum_{i=0}^{j} \delta z_{i}=a j+a \sum_{i=0}^{j} \zeta_{i}
$$

The resulting random perturbation of the atomic lattice is sketched in the fifth row of Fig. 3 and again this would entail a phase mismatch in the form

$$
\phi_{d_{j}}^{(c)}=\phi_{d}-\frac{\pi}{a} z_{j}^{(c)}=\phi_{d}-\pi \sum_{i=0}^{j} \zeta_{i} .
$$

This self-correlated disorder which takes into account the cumulative effect of layer-width fluctuations is used here as a simple characterization of solid layered media such as dielectric Bragg mirrors obtained by bottom-up epitaxy where it is challenging to keep a tight control over the layer thickness for a long growth time [59]. To this extent, it is also worth noticing that the atomic crystal setup described in Fig. 1 can be easily adapted to describe solid crystal setups where atoms are replaced by multilevel defect centers or impurities such as nitrogen-vacancy (NV) diamond or rare-earth-doped crystals [60].

\section{The numerical implementation}

Uncorrelated disorder algorithm. We consider a sample of, say, 20 disorder realizations for a cold-atomic lattice of length $L=M a$, with $M \gg 1$ being the number of periods. This is done by defining 20 arrays $R_{k}\{1, M\}(k=1,2, \ldots, 20)$ to store the random numbers uniformly distributed in the range of $\{-0.5,+0.5\}$. After $R_{k}(0), \ldots, R_{k}(j), \ldots, R_{k}(M)$ have been assigned different random values, we start to evaluate the averaged scattering properties of a probe field incident upon this sample by implementing each iteration of the 20 disorder realizations. Relevant results are shown in Figs. 4-13 by varying the dynamic level shift $\delta_{d 0}$ in the range of $\{-4.5$, $+4.5 \mathrm{MHz}\}$ when other parameters are fixed. In the first iteration, e.g., after calling $R_{1}(j)$ to define the disordered parameters $d_{j}$ or $z_{j}$, we can adopt the usual numerical approach to evaluate first the transfer matrix of the $j$ th period, then the transfer matrix of the whole sample, and finally the scattering properties. Similarly, we may call $R_{2}(j)$ to define the second disorder realization, and so on for the remaining realizations. Details for the case of uncorrelated geometric disorder are shown in Fig. 4. Once all 20 realizations have been obtained, a straightforward arithmetic mean procedure leads to the averaged quantities $R_{L}, R_{R}, \lambda_{s}^{+}$, and $\lambda_{s}^{-}$.

Self-correlated disorder algorithm. This type of disorder is studied using the same approach as above with the only difference lying in the fact that we redefine here $R_{k}(j+1)=$ $R_{k}(j)+C_{j+1}$, with $C_{j+1}$ being the randomly called numbers uniformly distributed in the range of $\{-0.5,+0.5\}$. We label with $k=1,2, \ldots, 20$ and $j=0,2, \ldots, M$ again, respectively, the iterations and the unit cells. An example for the case of self-correlated geometric disorder is shown in Fig. 5.

It is worth noting that although, in the two algorithms above, the random numbers are chosen to be uniformly distributed in the range of $\{-0.5,+0.5\}$ with a standard deviation $\sim 0.29$, nonuniform distributions may also be adopted. For a Gaussian distribution, e.g., with a half width $\sim 0.34$ corresponding to the same standard deviation, all results turn out to be basically the same as those obtained with the uniform distribution. 

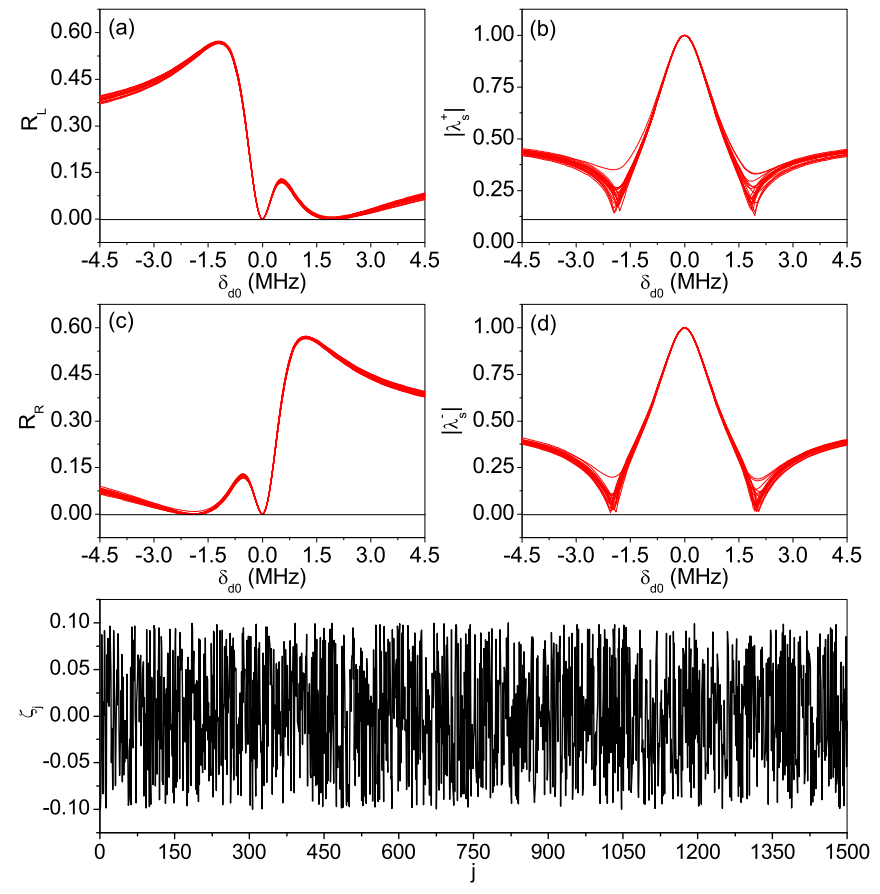

FIG. 4. Uncorrelated geometric disorder (site position). Top panels: Effect of uncorrelated disorder in the site position $z_{j}$ accompanied by the corresponding variation of phase mismatch $\phi_{d_{j}}$ [Eqs. (9) and (10)] on (a, c) probe reflectivities $R_{L}$ and $R_{R}$ as well as on (b, d) the moduli of scattering eigenvalues $\left|\lambda_{s}^{+}\right|$and $\left|\lambda_{s}^{-}\right|$. Each curve represents a different random configuration of disorder with maximum value $|\Delta a|=0.1 a$. Parameters are same as in Figs. 2(c) and 2(d), except $\Delta_{p}=0$ and $\lambda_{p} / \cos \theta_{p}=800.00 \mathrm{~nm}$. Bottom panel: An example of random-number sequence [Eq. (9)] leading to one of the 20 configurations of uncorrelated disorder.

\section{RESULTS AND DISCUSSION}

We examine in Fig. 6 the effects on URL and CPA of the uncorrelated disorder in the width $d$ of a Gaussian density profile [see Eq. (7)] as computed in Sec. II C for the lossy atomic lattice of Fig. 1(a). We show the reflectivities $R_{L}$ and $R_{R}$, respectively, in Figs. 6(a) and 6(c), while the eigenvalues $\left|\lambda_{s}^{+}\right|$and $\left|\lambda_{s}^{-}\right|$, respectively, are shown in Figs. 6(b) and 6(d). These panels indicate that both URL (the zeros of $R_{L}$ or $R_{R}$ at $\left|\delta_{d 0}\right| \approx 2.7 \mathrm{MHz}$ ) and CPA (the zeros of $\left|\lambda_{s}^{-}\right|$at $\left|\delta_{d 0}\right| \approx 3.0 \mathrm{MHz}$ ) points are rather robust against uncorrelated random variations $\left(\delta d_{j}\right)$ of $d$. However, we should note that both URL and, to a larger degree, CPA points slightly move towards higher $\left|\delta_{d 0}\right|$ values as the maximum amount of disorder $(\Delta d)$ increases. The trivial reflectivity zeros $R_{R}=R_{L}=0$ always appearing at $\delta_{d 0}=0$ for $\Delta_{c}=\Delta_{p}=0$ are, however, not affected by any kinds of disorder. URL and CPA points may also be found [44] for $\phi_{d} \neq \pi / 4$ and $\Delta_{p} \neq 0$, in which case our system will no longer be $P T$-antisymmetric or pseudo-Hermitian [49]. We examine in Fig. 7 the effects of uncorrelated disorder in the width $d$ on URL and CPA for a representative value of $\phi_{d}=0.15 \pi$ with $\Delta_{p}=1.363 \mathrm{MHz}$ [Figs. 7(a) and 7(c)] and $\Delta_{p}=1.525 \mathrm{MHz}$ [Figs. 7(b) and $7(\mathrm{~d})]$. The symmetry around $\delta_{d 0}=0$ is clearly lifted with disorder-induced modifications similar to that in Fig. 6.
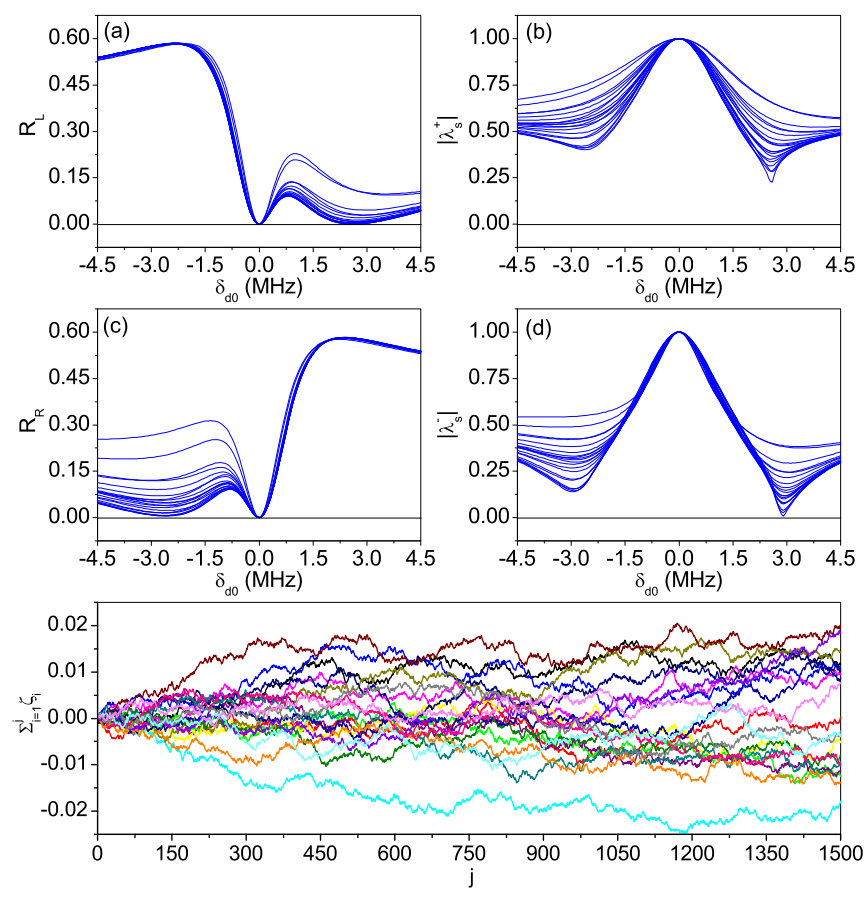

FIG. 5. Self-correlated geometric disorder (site position). Top panels: Effect of self-correlated disorder in the site position $z_{j}^{(c)}$ accompanied by the corresponding variation of phase mismatch $\phi_{d_{j}}^{(c)}$ [Eqs. (11) and (12)]. Each curve represents a different random configuration of disorder with maximum value $|\Delta a|=0.001 a$. Parameters are the same as in Figs. 2(c) and 2(d), except $\Delta_{p}=0$ and $\lambda_{p} / \cos \theta_{p}=$ $800.00 \mathrm{~nm}$. Bottom panel: An example of 20 random configurations of self-correlated disorder [Eq. (11)] exhibiting maximal absolute values $\sim 0.02 a$, which are much larger than $|\Delta a|$.
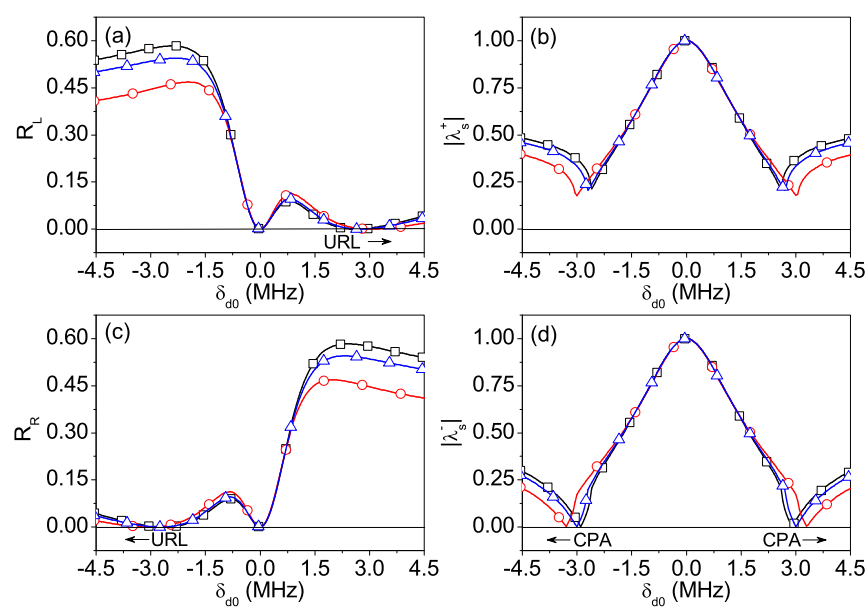

FIG. 6. Uncorrelated structural disorder (distribution width). (a, c) Probe reflectivities and $(b, d)$ scattering eigenvalues against maximal dynamic shift $\delta_{d 0}$ for uncorrelated disorder in the distribution widths $d_{j}$ [Eq. (7)]. Maximum disorder cases with $|\Delta d|=0.5 d$ (bluetriangle curves) and $|\Delta d|=1.0 d$ (red-circle curves) are compared to the ideal case (black-square curves) without disorder [2,44]. Red-circle and blue-triangle curves are attained through averaging over 20 different random configurations of disorder. Parameters are the same as in Figs. 4 and 5. 

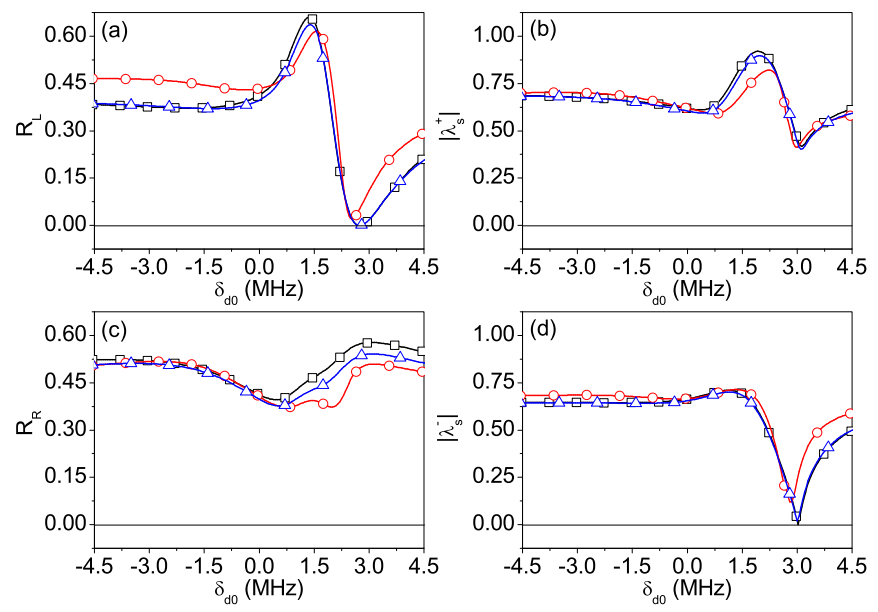

FIG. 7. Same as in Fig. 6, except $\phi_{d}=0.15 \pi$, (a, c) $\Delta_{p}=$ $1.363 \mathrm{MHz}$, and (b, d) $\Delta_{p}=1.525 \mathrm{MHz}$, a choice which lifts [44] the $P T$-antisymmetry and pseudo-Hermiticity (see Sec. II A).

In Fig. 8, we examine the effects of uncorrelated disorder in the site position $z_{j}$ [see Eq. (9)], accompanied by the corresponding variation of the phase mismatch $\phi_{d_{j}}$. It is to be noticed from comparing Fig. 6 with Fig. 8 that reflectivities $R_{L}$ and $R_{R}$ as well as eigenvalues $\left|\lambda_{s}^{+}\right|$and $\left|\lambda_{s}^{-}\right|$are modified differently with respect to the structural and geometric disorders, being in general much more sensitive to the latter than to the former. Increasing degrees of structural disorder, even orders of magnitude larger than those for the geometric case, do not destroy the crystal which remains periodic on average, hence exhibiting still efficient reflectivity resonances (Bragg peaks). In the case of geometric disorder, already a few percent level
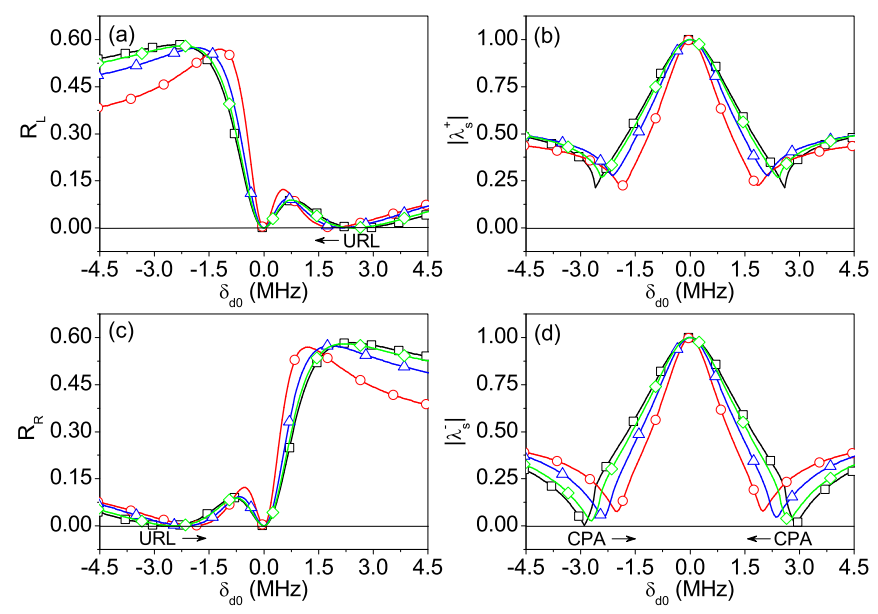

FIG. 8. Uncorrelated geometric disorder (site position). (a, c) Probe reflectivities and (b, d) scattering eigenvalues against maximal dynamic shift $\delta_{d 0}$ for uncorrelated disorder in the site position $z_{j}$ accompanied by the corresponding variation of the phase mismatch $\phi_{d_{j}}$ [Eqs. (9) and (10)]. Maximum disorder cases with $|\Delta a|=$ $0.05 a$ (green-diamond curve), $|\Delta a|=0.1 a$ (blue-triangle curve), and $|\Delta a|=0.2 a$ (red-circle curve) are compared to the ideal case (blacksquare curve) without disorder [2,44]. Red-circle, blue-triangle, and green-diamond curves are attained through averaging over 20 different random configurations of disorder. Parameters are the same as in Figs. 4 and 5.
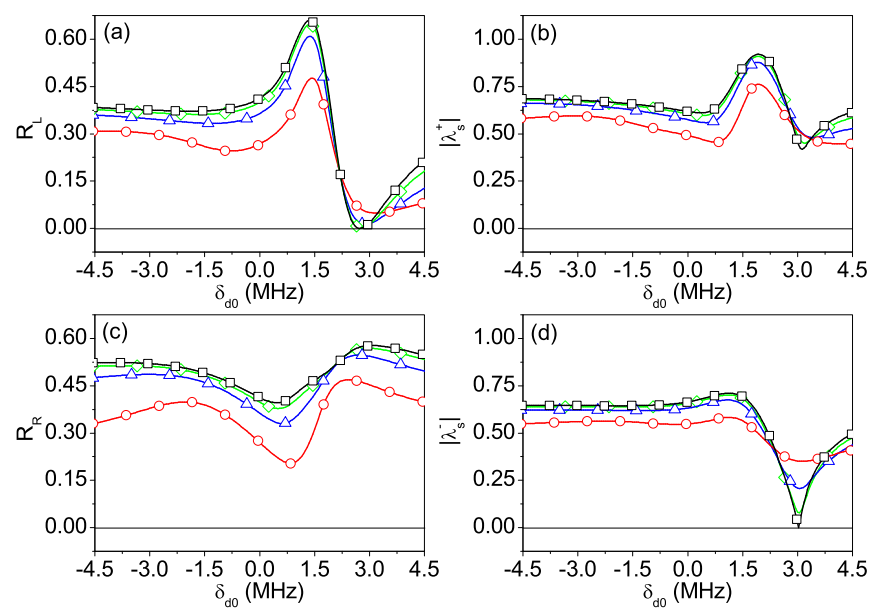

FIG. 9. Same as in Fig. 8, except $\phi_{d}=0.15 \pi$, (a, c) $\Delta_{p}=$ $1.363 \mathrm{MHz}$, and (b, d) $\Delta_{p}=1.525 \mathrm{MHz}$, a choice which lifts [44] the $P T$-antisymmetry and pseudo-Hermiticity (see Sec. II A).

of disorder changes the optical response by shifting, on the one hand, the URL position with respect to the ideal case and by making, on the other hand, the CPA point disappear. Somewhat larger disorder effects are observed in Fig. 9 where the case of $\phi_{d}=0.15 \pi$ is considered for $\Delta_{p}=1.363 \mathrm{MHz}$ [Figs. 9(a) and 9(c)] and $\Delta_{p}=1.525 \mathrm{MHz}$ [Figs. 9(b) and 9(d)]. Different effects of structural and geometric disorders are often found in various kinds of otherwise periodic systems, including photonic crystals [61-67]. As a matter of fact, geometric disorder is especially detrimental to our setup as it implies a concomitant variation in the phase mismatch between atomic distribution $\mathcal{N}_{j}(z)$ and dynamic shift $\delta_{d s}(z)$; this point is clearly confirmed in Fig. 10 where the phase mismatch has been artificially set equal to its ideal lattice value $\left(\phi_{d_{j}} \equiv \phi_{d}\right)$ (no cross correlation between $\phi_{d_{j}}$ and $z_{j}$ ). A comparison of Fig. 8 with Fig. 10 suggests that only for moderate amounts of uncorrelated geometric disorder both URL and CPA points remain, whereas for increasing disorder strengths, it is the
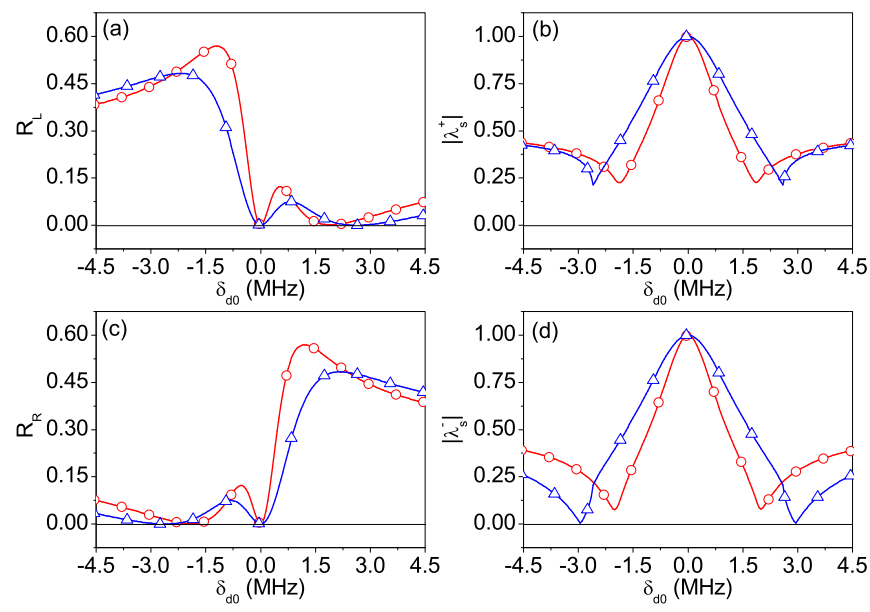

FIG. 10. Same as in Fig. 8 where results (red-circle curve) for uncorrelated disorder [Eqs. (9) and (10)] are compared here to results obtained with an artificial constant value of the phase mismatch $\phi_{d_{j}} \equiv \phi_{d}=\pi / 4$ (blue-triangle curve). Maximum disorder is kept at $|\Delta a|=0.2 a$ for both red-circle and blue-triangle curves. 

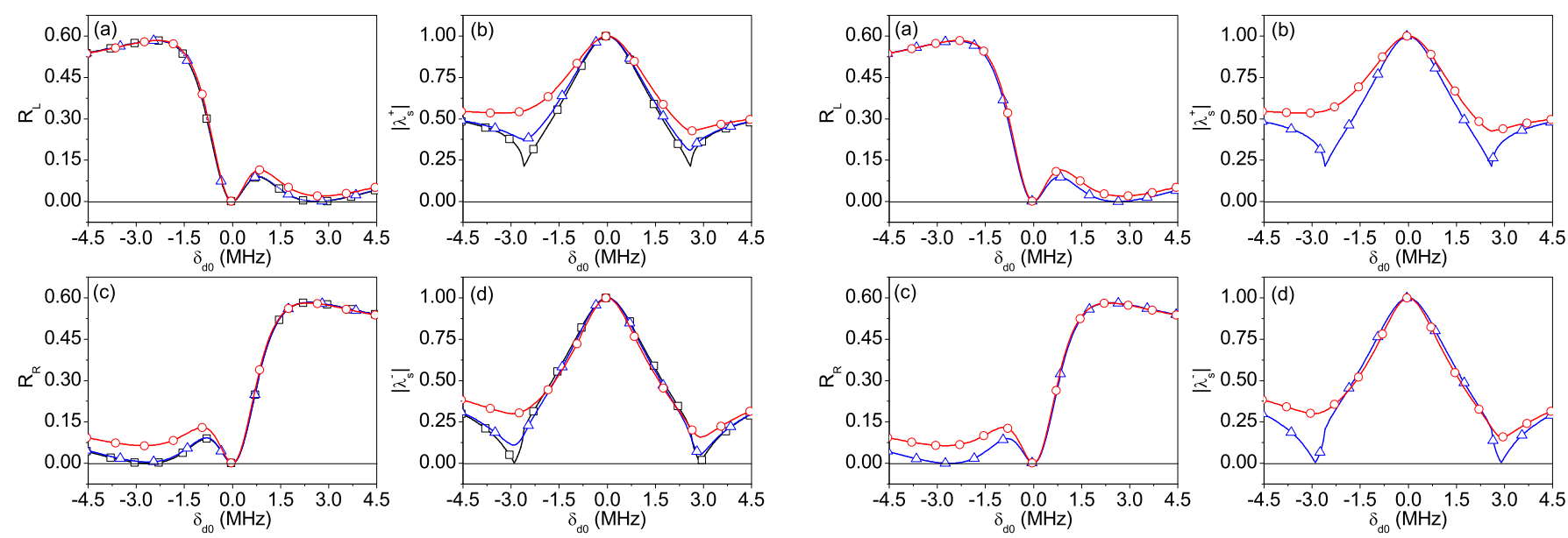

FIG. 11. Self-correlated geometric disorder (site position). (a, c) Probe reflectivities and (b, d) scattering eigenvalues against maximal dynamic shift $\delta_{d 0}$ for self-correlated disorder in the site position $z_{j}^{(c)}$ accompanied by the corresponding variation of the phase mismatch $\phi_{d_{j}}^{(c)}$ [Eqs. (11) and (12)]. Maximum disorder cases with $|\Delta a|=2.5 \times 10^{-4} a$ (blue-triangle curve) and $|\Delta a|=1.0 \times 10^{-3} a$ (red-circle curve) are compared to the ideal case (black-square curve) without disorder [2,44]. Red-circle and blue-triangle curves are attained through averaging over 20 different random configurations of disorder. Parameters are the same as in Figs. 4 and 5.

disorder-induced phase mismatch that mostly hampers the position where URL is to be observed and the occurrence of the CPA effect.

Finally, we consider the model of self-correlated geometric disorder described by $z_{j}^{(c)}$ [see Eq. (11)] with the concomitant phase mismatch $\phi_{d_{j}}^{(c)}$. Such a cumulative disorder is fairly common, e.g., in solids, where epitaxially grown layers are subject to fluctuations intrinsic to the growth process. This kind of disorder has the most dramatic effect on our photonic atomic crystal URL and CPA points as in Fig. 11; already very small percent fractions $(0.025 \%)$ in the period fluctuations lead to remarkable weakening of the URL and CPA effects. A similar behavior is also observed when we use different values
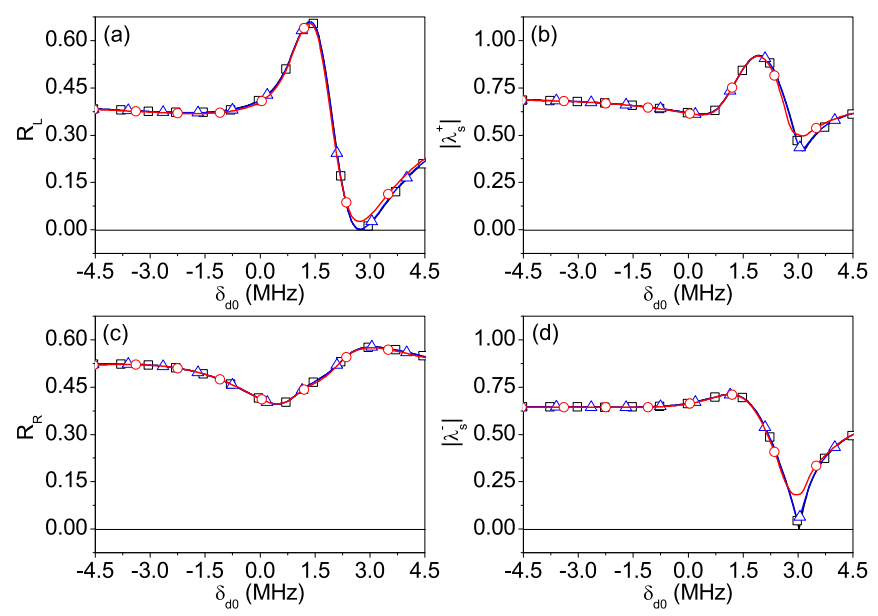

FIG. 12. Same as in Fig. 11, except $\phi_{d}=0.15 \pi$, (a, c) $\Delta_{p}=$ $1.363 \mathrm{MHz}$, and (b, d) $\Delta_{p}=1.525 \mathrm{MHz}$, a choice which lifts [44] the $P T$-antisymmetry and pseudo-Hermiticity (see Sec. II A).
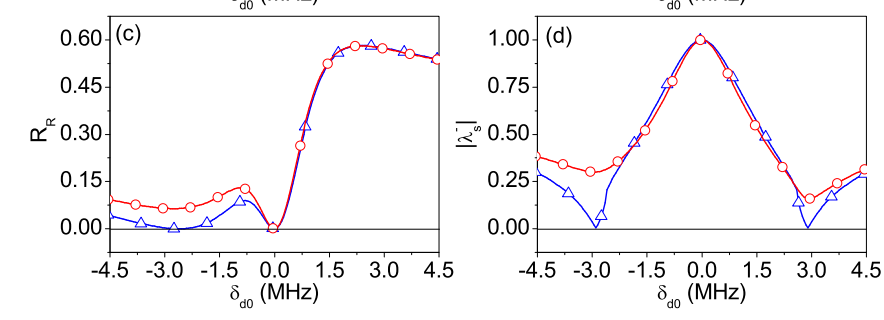

FIG. 13. Same as in Fig. 11, where results (red-circle curve) for self-correlated disorder [Eqs. (11) and (12)] are compared here to results obtained with an artificial constant value of the phase mismatch $\phi_{d_{j}}^{(c)} \equiv \phi_{d}^{(c)}=\pi / 4$ (blue-triangle curve). Maximum disorder is kept at $|\Delta a|=10^{-3} a$ for both red-circle and blue-triangle curves.

of the phase shift $\phi_{d}$ and the probe detuning $\Delta_{p}$ as in Fig. 12. Again it is the disorder-induced phase mismatch that largely blurs the URL and CPA points as is apparent from comparing Fig. 11 with Fig. 13.

\section{CONCLUSIONS}

At variance with standard solid photonic crystal structures, atomic photonic crystal setups [32,33,38-41] enable alloptical control of variant disorders $[29,69,70]$. Here we have addressed the issue of how robust are URL and CPA against uncorrelated and self-correlated disorders of familiar structural and geometric parameters, in a realistic photonic crystal structure obtained by driving cold atoms in an optical lattice to a multilevel EIT configuration [30-33,35-42]. We find that both URL and CPA are generally robust against structural disorder, though they appear rather sensitive against geometric disorder, mainly due to a concomitant variation in the phase mismatch between the cold-atomic density distributions and the dressing field spatial profiles. In particular, in the case of self-correlated disorder in the site position, CPA seems to disappear faster than URL, at least for the disorder strengths considered here. Needless to say, these strengths largely exceed those observed in typical optical lattice experiments, which conversely makes cold atoms loaded into optical lattices a system of choice to attain URL and CPA regimes. In fact, if disorder is not purposely introduced, e.g., via an additional speckle potential $[58,68]$, the optical lattice periodicity is practically ideal, as only randomness in the number of cold atoms loaded into each well is, in general, present. To this purpose, we have also ascertained the effect of random fluctuations $\delta N_{j}$ in the atomic density $N$ and found that such a source of structural disorder entails changes in the reflectivities $\left(R_{L}\right.$ and $\left.R_{R}\right)$ and the eigenvalues $\left(\left|\lambda_{s}^{+}\right|\right.$and $\left.\left|\lambda_{s}^{-}\right|\right)$that amount to a tiny fraction of percents $(\sim 0.2 \%)$ of the changes one would observe, e.g., for disorder in the distribution width $d$ (Figs. 6 and 7). 
Finally, we note that our results may also be relevant to acoustic waves in layered media owing to the fact that acoustic and optical waves obey similar propagation equations [71-74]. To this extent, the realization of a coherently tunable and periodically modulated acoustic response may turn out to be an attractive prospect for acoustic metamaterials [75-79].

\section{ACKNOWLEDGMENTS}

We are grateful to F. Scazza for enlightening discussions on issues of disorder. J.-H.W. would like to thank the Scuola Normale Superiore at Pisa for the hospitality. The work is supported by the National Natural Science Foundation (Grants No. 61378094, No. 10534002, and No. 11674049) of China.
[1] L. Feng, Y.-L. Xu, W. S. Fegadolli, M.-H. Lu, J. E. B. Oliveira, V. R. Almeida, Y.-F. Chen, and A. Scherer, Nat. Mater. 12, 108 (2012).

[2] J.-H. Wu, M. Artoni, and G. C. La Rocca, Phys. Rev. Lett. 113, 123004 (2014).

[3] S. A. R. Horsley, M. Artoni, and G. C. La Rocca, Nat. Photon. 9, 436 (2015).

[4] Y.-D. Chong, L. Ge, H. Cao, and A. D. Stone, Phys. Rev. Lett. 105, 053901 (2010).

[5] S. Longhi, Phys. Rev. A 82, 031801(R) (2010).

[6] J. Zhang, K. F. MacDonald, and N. I. Zheludev, Light: Sci. Appl. 1, e18 (2012).

[7] X. Fang, M. L. Tseng, J.-Y. Ou, K. F. MacDonald, D. P. Tsai, and N. I. Zheludev, Appl. Phys. Lett. 104, 141102 (2014).

[8] Y. Sun, W. Tan, H.-Q. Li, J. Li, and H. Chen, Phys. Rev. Lett. 112, 143903 (2014).

[9] S. Dutta-Gupta, O. J. F. Martin, S. Dutta Gupta, and G. S. Agarwal, Opt. Express 20, 1330 (2012).

[10] W. Wan, Y. Chong, L. Ge, H. Noh, A. D. Stone, and H. Cao, Science 331, 889 (2011).

[11] S. Longhi, Phys. Rev. A 83, 055804 (2011).

[12] S. Feng and K. Halterman, Phys. Rev. B 86, 165103 (2012).

[13] H. Noh, Y. Chong, A. D. Stone, and H. Cao, Phys. Rev. Lett. 108, 186805 (2012).

[14] M. Pu, Q. Feng, M. Wang, C. Hu, C. Huang, X. Ma, Z. Zhao, C. Wang, and X. Luo, Opt. Express 20, 2246 (2012).

[15] N. Gutman, A. A. Sukhorukov, Y. Chong, and C. M. de Sterke, Opt. Lett. 38, 4970 (2013).

[16] R. Bruck and O. L. Muskens, Opt. Express 21, 27652 (2013).

[17] J.-F. Zhang, C.-C. Guo, K. Liu, Z.-H. Zhu, W.-M. Ye, X.-D. Yuan, and S.-Q. Qin, Opt. Express 22, 12524 (2014).

[18] T. Kottos, Nat. Phys. 6, 166 (2010).

[19] J. D. Joannopoulos, S. G. Johnson, J. N. Winn, and R. D. Meade, in Photonic Crystals: Molding the Flow of Light, 2nd ed. (Princeton University Press, Princeton, 2008).

[20] M. Born and E. Wolf, Principles of Optics (Cambridge University Press, Cambridge, 1999).

[21] I. Bloch, Nat. Phys. 1, 23 (2005).

[22] S. A. R. Horsley, J.-H. Wu, M. Artoni, and G. C. La Rocca, Phys. Rev. Lett. 110, 223602 (2013).

[23] M. Greiner, O. Mandel, T. Esslinger, T. W. Hansch, and I. Bloch, Nature (London) 415, 39 (2002).

[24] B. Paredes, A. Widera, V. Murg, O. Mandel, S. Folling, I. Cirac, G. V. Shlyapnikov, T. W. Hansch, and I. Bloch, Nature (London) 429, 277 (2004).

[25] T. Kinoshita, T. Wenger, and D. S. Weiss, Science 305, 1125 (2004).

[26] I. Bloch, Phys. World 17, 25 (2004).
[27] I. Bloch, J. Dalibard, and W. Zwerger, Rev. Mod. Phys. 80, 885 (2008).

[28] M. Lewenstein, A. Sanpera, and V. Ahufinger, in Ultracold Atoms in Optical Lattices. Simulating Quantum Many-Body Systems (Oxford University Press, New York, 2012).

[29] M. Lewenstein, A. Sanpera, V. Ahufinger, B. Damski, A. De Sen, and U. Sen, Adv. Phys. 56, 243 (2007).

[30] G. Birkl, M. Gatzke, I. H. Deutsch, S. L. Rolston, and W. D. Phillips, Phys. Rev. Lett. 75, 2823 (1995).

[31] M. Weidemuller, A. Hemmerich, A. Gorlitz, T. Esslinger, and T. W. Hansch, Phys. Rev. Lett. 75, 4583 (1995).

[32] M. Artoni and G. C. La Rocca, Phys. Rev. Lett. 96, 073905 (2006).

[33] A. Schilke, C. Zimmermann, P. W. Courteille, and W. Guerin, Phys. Rev. Lett. 106, 223903 (2011).

[34] S. A. R. Horsley, M. Artoni, and G. C. La Rocca, Phys. Rev. Lett. 107, 043602 (2011).

[35] A. Schilke, C. Zimmermann, P. W. Courteille, and W. Guerin, Nat. Photon. 6, 101 (2012).

[36] D.-S. Yu, Phys. Rev. A 84, 043833 (2011).

[37] A. Schilke, C. Zimmermann, and W. Guerin, Phys. Rev. A 86, 023809 (2012).

[38] J.-H. Wu, M. Artoni, and G. C. La Rocca, Phys. Rev. A 88, 043823 (2013).

[39] Y.-Q. Zhang, Z.-K. Wu, X. Yao, Z.-Y. Zhang, H.-X. Chen, H.-B. Zhang, and Y.-P. Zhang, Opt. Express 21, 29338 (2013).

[40] M.-Q. Gao, Z.-G. Wang, Z. Ullah, H.-X. Chen, D. Zhang, Y.-Q. Zhang, and Y.-P. Zhang, J. Opt. Soc. Am. B 32, 179 (2015).

[41] Z.-G. Wang, Z. Ullah, M.-Q. Gao, D. Zhang, Y.-Q. Zhang, H. Gao, and Y.-P. Zhang, Sci. Rep. 5, 13880 (2015).

[42] Z.-G. Wang, M.-Q. Gao, A. R. Mahesar, and Y.-P. Zhang, Sci. Rep. 6, 28185 (2016).

[43] J.-H. Wu, M. Artoni, and G. C. La Rocca, Phys. Rev. A 91, 033811 (2015).

[44] J.-H. Wu, M. Artoni, and G. C. La Rocca, Sci. Rep. 6, 35356 (2016)

[45] S. F. Liew and H. Cao, J. Opt. 12, 024011 (2010).

[46] A. Mostafazadeh, Int. J. Geom. Methods Mod. Phys. 07, 1191 (2010).

[47] M. Artoni, G. C. La Rocca, and F. Bassani, Phys. Rev. E 72, 046604 (2005)

[48] M. Fleischhauer, A. Imamoglu, and J. P. Marangos, Rev. Mod. Phys. 77, 633 (2005)

[49] A. Mostafazadeh, Phys. Rev. Lett. 102, 220402 (2009).

[50] M. Berry, Czech. J. Phys. 54, 1039 (2004).

[51] W. D. Heiss, J. Phys. A 45, 444016 (2012).

[52] T. Kato, in Perturbation Theory of Linear Operators (Springer, Berlin, 1966). 
[53] R. B. Diener, G. A. Georgakis, J. Zhong, M. Raizen, and Q. Niu, Phys. Rev. A 64, 033416 (2001).

[54] M. White, M. Pasienski, D. McKay, S. Q. Zhou, D. Ceperley, and B. DeMarco, Phys. Rev. Lett. 102, 055301 (2009).

[55] W. R. McGehee, S. S. Kondov, W. Xu, J. J. Zirbel, and B. DeMarco, Phys. Rev. Lett. 111, 145303 (2013).

[56] Y.-S. Cheng and S. K. Adhikari, Phys. Rev. A 82, 013631 (2010).

[57] D. Delande and G. Orso, Phys. Rev. Lett. 113, 060601 (2014).

[58] G. Modugno, Rep. Prog. Phys. 73, 102401 (2010).

[59] A. Bhattacharya, M. Nasarek, U. Zeimer, A. Klein, M. Zorn, F. Bugge, S. Gramlich, and M. Weyers, J. Cryst. Growth 274, 331 (2005).

[60] Q.-Y. He, Y. Xue, M. Artoni, G. C. La Rocca, J.-H. Xu, and J.-Y. Gao, Phys. Rev. B 73, 195124 (2006).

[61] V. D. Freilikher, B. A. Liansky, I. V. Yurkevich, A. A. Maradudin, and A. R. McGurn, Phys. Rev. E 51, 6301 (1995).

[62] Y. A. Vlasov, M. I. Kaliteevski, and V. V. Nikolaev, Phys. Rev. B 60, 1555 (1999).

[63] Z.-Y. Li and Z.-Q. Zhang, Phys. Rev. B 62, 1516 (2000).

[64] A. F. Koenderink, A. Lagendijk, and W. L. Vos, Phys. Rev. B 72, 153102 (2005).

[65] R. Rengarajan, D. Mittleman, C. Rich, and V. Colvin, Phys. Rev. E 71, 016615 (2005).

[66] A. Maurel and P. A. Martin, Eur. Phys. J. B 86, 486 (2013).
[67] A. N. Poddubny, M. V. Rybin, M. F. Limonov, and Y. S. Kivshar, Nat. Commun. 3, 914 (2012).

[68] B. Shapiro, J. Phys. A 45, 143001 (2012).

[69] L. Sanchez-Palencia and M. Lewenstein, Nat. Phys. 6, 87 (2010).

[70] M. Pasienski, D. McKay, M. White, and B. DeMarco, Nat. Phys. 6, 677 (2010)

[71] A. Esmailpour, M. Esmailpour, A. Sheikhan, M. Elahi, M. R. Tabar, and M. Sahimi, Phys. Rev. B 78, 134206 (2008).

[72] A. Khelif, Y. Achaoui, S. Benchabane, V. Laude, and B. Aoubiza, Phys. Rev. B 81, 214303 (2010).

[73] J. Christensen and F. J. G. de Abajo, Phys. Rev. B 86, 024301 (2012).

[74] A. Khelif and A. Adibi, Phononic Crystals: Fundamentals and Applications (Springer, New York, 2015).

[75] F. Liu, M. Ke, A. Zhang, W. Wen, J. Shi, Z. Liu, and P. Sheng, Phys. Rev. E 82, 026601 (2010).

[76] X.-F. Zhu, H. Ramezani, C.-Z. Shi, J. Zhu, and X. Zhang, Phys. Rev. X 4, 031042 (2014).

[77] J. A. Souza, L. Cabral, R. R. Oliveira, and C. J. Villas-Boas, Phys. Rev. A 92, 023818 (2015).

[78] S. A. Cummer, J. Christensen, and A. Alù, Nat. Rev. Mater. 1, 16001 (2016)

[79] G. Ma and P. Sheng, Sci. Adv. 2, 1501595 (2016). 